\title{
INEQUALITIES INVOLVING THE MEAN AND \\ THE STANDARD DEVIATION OF NONNEGATIVE \\ REAL NUMBERS
}

OSCAR ROJO

Received 22 December 2005; Revised 18 August 2006; Accepted 21 September 2006

Let $m(\mathbf{y})=\sum_{j=1}^{n} y_{j} / n$ and $s(\mathbf{y})=\sqrt{m\left(\mathbf{y}^{2}\right)-m^{2}(\mathbf{y})}$ be the mean and the standard deviation of the components of the vector $\mathbf{y}=\left(y_{1}, y_{2}, \ldots, y_{n-1}, y_{n}\right)$, where $\mathbf{y}^{q}=\left(y_{1}^{q}, y_{2}^{q}, \ldots, y_{n-1}^{q}, y_{n}^{q}\right)$ with $q$ a positive integer. Here, we prove that if $\mathbf{y} \geq \mathbf{0}$, then $m\left(\mathbf{y}^{2^{p}}\right)+(1 / \sqrt{n-1}) s\left(\mathbf{y}^{2^{p}}\right) \leq$ $\sqrt{m\left(\mathbf{y}^{2^{p+1}}\right)+(1 / \sqrt{n-1}) s\left(\mathbf{y}^{2^{p+1}}\right)}$ for $p=0,1,2, \ldots$ The equality holds if and only if the $(n-1)$ largest components of $\mathbf{y}$ are equal. It follows that $\left(l_{2^{p}}(\mathbf{y})\right)_{p=0}^{\infty}, l_{2^{p}}(\mathbf{y})=$ $\left(m\left(\mathbf{y}^{2^{p}}\right)+(1 / \sqrt{n-1}) s\left(\mathbf{y}^{2^{p}}\right)\right)^{2^{-p}}$, is a strictly increasing sequence converging to $y_{1}$, the largest component of $\mathbf{y}$, except if the $(n-1)$ largest components of $\mathbf{y}$ are equal. In this case, $l_{2^{p}}(\mathbf{y})=y_{1}$ for all $p$.

Copyright (c) 2006 Oscar Rojo. This is an open access article distributed under the Creative Commons Attribution License, which permits unrestricted use, distribution, and reproduction in any medium, provided the original work is properly cited.

\section{Introduction}

Let

$$
m(\mathbf{x})=\frac{\sum_{j=1}^{n} x_{j}}{n}, \quad s(\mathbf{x})=\sqrt{m\left(\mathbf{x}^{2}\right)-m^{2}(\mathbf{x})}
$$

be the mean and the standard deviation of the components of $\mathbf{x}=\left(x_{1}, x_{2}, \ldots, x_{n-1}, x_{n}\right)$, where $\mathbf{x}^{q}=\left(x_{1}^{q}, x_{2}^{q}, \ldots, x_{n-1}^{q}, x_{n}^{q}\right)$ for a positive integer $q$.

The following theorem is due to Wolkowicz and Styan [3, Theorem 2.1.].

Theorem 1.1. Let

$$
x_{1} \geq x_{2} \geq \cdots \geq x_{n-1} \geq x_{n} .
$$


2 Inequalities on the mean and standard deviation

Then

$$
\begin{aligned}
& m(\mathbf{x})+\frac{1}{\sqrt{n-1}} s(\mathbf{x}) \leq x_{1}, \\
& x_{1} \leq m(\mathbf{x})+\sqrt{n-1} s(\mathbf{x}) .
\end{aligned}
$$

Equality holds in (1.3) if and only if $x_{1}=x_{2}=\cdots=x_{n-1}$. Equality holds in (1.4) if and only if $x_{2}=x_{3}=\cdots=x_{n}$.

Let $x_{1}, x_{2}, \ldots, x_{n-1}, x_{n}$ be complex numbers such that $x_{1}$ is a positive real number and

$$
x_{1} \geq\left|x_{2}\right| \geq \cdots \geq\left|x_{n-1}\right| \geq\left|x_{n}\right| \text {. }
$$

Then,

$$
x_{1}^{p} \geq\left|x_{2}\right|^{p} \geq \cdots \geq\left|x_{n-1}\right|^{p} \geq\left|x_{n}\right|^{p}
$$

for any positive integer $p$. We apply Theorem 1.1 to (1.6) to obtain

$$
\begin{aligned}
& m\left(|\mathbf{x}|^{p}\right)+\frac{1}{\sqrt{n-1}} s\left(|\mathbf{x}|^{p}\right) \leq x_{1}^{p}, \\
& x_{1}^{p} \leq m\left(|\mathbf{x}|^{p}\right)+\sqrt{n-1} s\left(|\mathbf{x}|^{p}\right),
\end{aligned}
$$

where $|\mathbf{x}|=\left(\left|x_{1}\right|,\left|x_{2}\right|, \ldots,\left|x_{n-1}\right|,\left|x_{n}\right|\right)$.

Then,

$$
l_{p}(\mathbf{x})=\left(m\left(|\mathbf{x}|^{p}\right)+\frac{1}{\sqrt{n-1}} s\left(|\mathbf{x}|^{p}\right)\right)^{1 / p}
$$

is a sequence of lower bounds for $x_{1}$ and

$$
u_{p}(\mathbf{x})=\left(m\left(|\mathbf{x}|^{p}\right)+\sqrt{n-1} s\left(|\mathbf{x}|^{p}\right)\right)^{1 / p}
$$

is a sequence of upper bounds for $x_{1}$.

We recall that the $p$-norm and the infinity-norm of a vector $\mathbf{x}=\left(x_{1}, x_{2}, \ldots, x_{n}\right)$ are

$$
\begin{gathered}
\|\mathbf{x}\|_{p}=\left(\sum_{i=1}^{n}\left|x_{i}\right|^{p}\right)^{1 / p}, \quad 1 \leq p<\infty, \\
\|\mathbf{x}\|_{\infty}=\max _{i}\left|x_{i}\right| .
\end{gathered}
$$

It is well known that $\lim _{p \rightarrow \infty}\|\mathbf{x}\|_{p}=\|\mathbf{x}\|_{\infty}$. 
Then,

$$
\begin{gathered}
l_{p}(\mathbf{x})=\left(\frac{\|\mathbf{x}\|_{p}^{p}}{n}+\frac{1}{\sqrt{n(n-1)}} \sqrt{\|\mathbf{x}\|_{2 p}^{2 p}-\frac{\|\mathbf{x}\|_{p}^{2 p}}{n}}\right)^{1 / p}, \\
u_{p}(\mathbf{x})=\left(\frac{\|\mathbf{x}\|_{p}^{p}}{n}+\sqrt{\frac{n-1}{n}} \sqrt{\|\mathbf{x}\|_{2 p}^{2 p}-\frac{\|\mathbf{x}\|_{p}^{2 p}}{n}}\right)^{1 / p} .
\end{gathered}
$$

In [2, Theorem 11], we proved that if $y_{1} \geq y_{2} \geq y_{3} \geq \cdots \geq y_{n} \geq 0$, then

$$
m\left(\mathbf{y}^{2^{p}}\right)+\sqrt{n-1} s\left(\mathbf{y}^{2^{p}}\right) \geq \sqrt{m\left(\mathbf{y}^{2^{p+1}}\right)+\sqrt{n-1} s\left(\mathbf{y}^{2^{p+1}}\right)}
$$

for $p=0,1,2, \ldots$. The equality holds if and only if $y_{2}=y_{3}=\cdots=y_{n}$. Using this inequality, we proved in [2, Theorems 14 and 15] that if $y_{2}=y_{3}=\cdots=y_{n}$, then $u_{p}(\mathbf{y})=y_{1}$ for all $p$, and if $y_{i}<y_{j}$ for some $2 \leq j<i \leq n$, then $\left(u_{2^{p}}(\mathbf{y})\right)_{p=0}^{\infty}$ is a strictly decreasing sequence converging to $y_{1}$.

The main purpose of this paper is to prove that if $y_{1} \geq y_{2} \geq y_{3} \geq \cdots \geq y_{n} \geq 0$, then

$$
m\left(\mathbf{y}^{2^{p}}\right)+\frac{1}{\sqrt{n-1}} s\left(\mathbf{y}^{2^{p}}\right) \leq \sqrt{m\left(\mathbf{y}^{2^{p+1}}\right)+\frac{1}{\sqrt{n-1}} s\left(\mathbf{y}^{2^{p+1}}\right)}
$$

for $p=0,1,2, \ldots$. The equality holds if and only if $y_{1}=y_{2}=\cdots=y_{n-1}$. Using this inequality, we prove that if $y_{1}=y_{2}=\cdots=y_{n-1}$, then $u_{p}(\mathbf{y})=y_{1}$ for all $p$, and if $y_{i}<y_{j}$ for some $1 \leq j<i \leq n-1$, then $\left(l_{2^{p}}(\mathbf{y})\right)_{p=0}^{\infty}$ is a strictly increasing sequence converging to $y_{1}$.

\section{New inequalities involving $m(\mathbf{x})$ and $s(\mathbf{x})$}

Theorem 2.1. Let $\mathbf{x}=\left(x_{1}, x_{2}, \ldots, x_{n-1}, x_{n}\right)$ be a vector of complex numbers such that $x_{1}$ is a positive real number and

$$
x_{1} \geq\left|x_{2}\right| \geq \cdots \geq\left|x_{n-1}\right| \geq\left|x_{n}\right| \text {. }
$$

The sequence $\left(l_{p}(\mathbf{x})\right)_{p=1}^{\infty}$ converges to $x_{1}$.

Proof. From (1.11),

$$
l_{p}(\mathbf{x}) \geq \frac{\|\mathbf{x}\|_{p}}{\sqrt[p]{n}} \quad \forall p
$$

Then, $0 \leq\left|l_{p}(\mathbf{x})-x_{1}\right|=x_{1}-l_{p}(\mathbf{x}) \leq x_{1}-\|\mathbf{x}\|_{p} / \sqrt[p]{n}$ for all $p$. Since $\lim _{p \rightarrow \infty}\|\mathbf{x}\|_{p}=x_{1}$ and $\lim _{p \rightarrow \infty} \sqrt[p]{n}=1$, it follows that the sequence $\left(l_{p}(\mathbf{x})\right)$ converges and $\lim _{p \rightarrow \infty} l_{p}(\mathbf{x})=x_{1}$.

We introduce the following notations:

(i) $\mathbf{e}=(1,1, \ldots, 1)$,

(ii) $\mathscr{D}=\mathbb{R}^{n}-\{\lambda \mathbf{e}: \lambda \in \mathbb{R}\}$,

(iii) $\mathscr{C}=\left\{\mathbf{x}=\left(x_{1}, x_{2}, \ldots, x_{n}\right): 0 \leq x_{k} \leq 1, k=1,2, \ldots, n\right\}$, 
4 Inequalities on the mean and standard deviation

(iv) $\mathscr{E}=\left\{\mathbf{x}=\left(1, x_{2}, \ldots, x_{n}\right): 0 \leq x_{n} \leq x_{n-1} \leq \cdots \leq x_{2} \leq 1\right\}$,

(v) $\langle\mathbf{x}, \mathbf{y}\rangle=\sum_{k=1}^{n} x_{k} y_{k}$ for $\mathbf{x}, \mathbf{y} \in \mathbb{R}^{n}$,

(vi) $\nabla g(\mathbf{x})=\left(\partial_{1} g(\mathbf{x}), \partial_{2} g(\mathbf{x}), \ldots, \partial_{n} g(\mathbf{x})\right)$ denotes the gradient of a differentiable function $g$ at the point $\mathbf{x}$, where $\partial_{k} g(\mathbf{x})$ is the partial derivative of $g$ with respect to $x_{k}$, evaluated at $\mathbf{x}$.

Clearly, if $\mathbf{x} \in \mathscr{E}$, then $\mathbf{x}^{q} \in \mathscr{E}$ with $q$ a positive integer.

Let $\mathbf{v}_{1}, \mathbf{v}_{2}, \ldots, \mathbf{v}_{n}$ be the points

$$
\begin{gathered}
\mathbf{v}_{1}=(1,0, \ldots, 0), \\
\mathbf{v}_{2}=(1,1,0, \ldots, 0), \\
\mathbf{v}_{3}=(1,1,1,0, \ldots, 0), \\
\vdots \\
\mathbf{v}_{n-2}=(1,1, \ldots, 1,0,0), \\
\mathbf{v}_{n-1}=(1,1, \ldots, 1,1,0), \\
\mathbf{v}_{n}=(1,1, \ldots, 1,1)=\mathbf{e} .
\end{gathered}
$$

Observe that $\mathbf{v}_{1}, \mathbf{v}_{2}, \ldots, \mathbf{v}_{n}$ lie in $\mathscr{E}$. For any $\mathbf{x}=\left(1, x_{2}, x_{3}, \ldots, x_{n-1}, x_{n}\right) \in \mathscr{E}$, we have

$$
\begin{aligned}
\mathbf{X}= & \left(1-x_{2}\right) \mathbf{v}_{1}+\left(x_{2}-x_{3}\right) \mathbf{v}_{2}+\left(x_{3}-x_{4}\right) \mathbf{v}_{3} \\
& +\cdots+\left(x_{n-2}-x_{n-1}\right) \mathbf{v}_{n-2}+\left(x_{n-1}-x_{n}\right) \mathbf{v}_{n-1}+x_{n} \mathbf{v}_{n} .
\end{aligned}
$$

Therefore, $\mathscr{E}$ is a convex set. We define the function

$$
f(\mathbf{x})=m(\mathbf{x})+\frac{1}{\sqrt{n-1}} s(\mathbf{x})
$$

where $\mathbf{x}=\left(x_{1}, x_{2}, \ldots, x_{n}\right) \in \mathbb{R}^{n}$. We observe that

$$
\begin{aligned}
n s^{2}(\mathbf{x}) & =\sum_{k=1}^{n} x_{k}^{2}-\frac{\left(\sum_{j=1}^{n} x_{j}\right)^{2}}{n}=\sum_{k=1}^{n}\left(x_{k}-m(\mathbf{x})\right)^{2} \\
& =\|\mathbf{x}-m(\mathbf{x}) \mathbf{e}\|_{2}^{2} .
\end{aligned}
$$

Then,

$$
\begin{aligned}
f(\mathbf{x}) & =m(\mathbf{x})+\frac{1}{\sqrt{n(n-1)}}\|\mathbf{x}-m(\mathbf{x}) \mathbf{e}\|_{2} \\
& =\frac{\sum_{j=1}^{n} x_{j}}{n}+\frac{1}{\sqrt{n(n-1)}} \sqrt{\sum_{k=1}^{n} x_{k}^{2}-\frac{\left(\sum_{j=1}^{n} x_{j}\right)^{2}}{n}} .
\end{aligned}
$$

Next, we give properties of $f$. Some of the proofs are similar to those in [2]. 
Lemma 2.2. The function $f$ has continuous first partial derivatives on $\mathscr{D}$, and for $\mathbf{x}=$ $\left(x_{1}, x_{2}, \ldots, x_{n}\right) \in \mathscr{D}$ and $1 \leq k \leq n$,

$$
\begin{gathered}
\partial_{k} f(\mathbf{x})=\frac{1}{n}+\frac{1}{n(n-1)} \frac{x_{k}-m(\mathbf{x})}{f(\mathbf{x})-m(\mathbf{x})}, \\
\sum_{k=1}^{n} \partial_{k} f(\mathbf{x})=1, \\
\langle\nabla f(\mathbf{x}), \mathbf{x}\rangle=f(\mathbf{x}) .
\end{gathered}
$$

Proof. From (2.7), it is clear that $f$ is differentiable at every point $\mathbf{x} \neq m(\mathbf{x}) \mathbf{e}$, and for $1 \leq k \leq n$,

$$
\begin{aligned}
\partial_{k} f(\mathbf{x}) & =\frac{1}{n}+\frac{1}{\sqrt{n(n-1)}} \frac{x_{k}-\sum_{j=1}^{n} x_{j} / n}{\sqrt{\sum_{i=1}^{n} x_{i}^{2}-\left(\sum_{j=1}^{n} x_{j}\right)^{2} / n}} \\
& =\frac{1}{n}+\frac{1}{n(n-1)} \frac{x_{k}-m(\mathbf{x})}{f(\mathbf{x})-m(\mathbf{x})},
\end{aligned}
$$

which is a continuous function on $\mathscr{D}$. Then, $\sum_{k=1}^{n} \partial_{k} f(\mathbf{x})=1$. Finally,

$$
\begin{aligned}
\langle\nabla f(\mathbf{x}), \mathbf{x}\rangle & =\sum_{k=1}^{n} x_{k} \partial_{k} f(\mathbf{x}) \\
& =\frac{\sum_{k=1}^{n} x_{k}}{n}+\frac{1}{n(n-1)} \frac{\sum_{k=1}^{n} x_{k}^{2}-m(\mathbf{x}) \sum_{k=1}^{n} x_{k}}{f(\mathbf{x})-m(\mathbf{x})} \\
& =m(\mathbf{x})+\frac{1}{\sqrt{n(n-1)}}\|\mathbf{x}-a(\mathbf{x}) \mathbf{e}\|_{2}=f(\mathbf{x}) .
\end{aligned}
$$

This completes the proof.

Lemma 2.3. The function $f$ is convex on $\mathscr{C}$. More precisely, for $\mathbf{x}, \mathbf{y} \in \mathscr{C}$ and $t \in[0,1]$,

$$
f((1-t) \mathbf{x}+t \mathbf{y}) \leq(1-t) f(\mathbf{x})+t f(\mathbf{y})
$$

with equality if and only if

$$
\mathbf{x}-m(\mathbf{x}) \mathbf{e}=\alpha(\mathbf{y}-m(\mathbf{y}) \mathbf{e})
$$

for some $\alpha \geq 0$.

Proof. Clearly $\mathscr{C}$ is a convex set. Let $\mathbf{x}, \mathbf{y} \in \mathscr{C}$ and $t \in[0,1]$. Then,

$$
\begin{aligned}
f((1-t) \mathbf{x}+t \mathbf{y}) & =m((1-t) \mathbf{x}+t \mathbf{y})+\frac{1}{\sqrt{n(n-1)}}\|(1-t) \mathbf{x}+t \mathbf{y}-m((1-t) \mathbf{x}+t \mathbf{y}) \mathbf{e}\|_{2} \\
& =(1-t) m(\mathbf{x})+t m(\mathbf{y})+\frac{1}{\sqrt{n(n-1)}}\|(1-t)(\mathbf{x}-m(\mathbf{x}) \mathbf{e})+t(\mathbf{y}-m(\mathbf{y}) \mathbf{e})\|_{2} .
\end{aligned}
$$


6 Inequalities on the mean and standard deviation

Moreover,

$$
\begin{aligned}
& \|(1-t)(\mathbf{x}-m(\mathbf{x}) \mathbf{e})+t(\mathbf{y}-m(\mathbf{y}) \mathbf{e})\|_{2}^{2} \\
& \quad=(1-t)^{2}\|\mathbf{x}-m(\mathbf{x}) \mathbf{e}\|_{2}^{2}+2(1-t) t\langle\mathbf{x}-m(\mathbf{x}) \mathbf{e}, \mathbf{y}-m(\mathbf{y}) \mathbf{e}\rangle+t^{2}\|\mathbf{y}-m(\mathbf{y}) \mathbf{e}\|_{2}^{2} .
\end{aligned}
$$

We recall the Cauchy-Schwarz inequality to obtain

$$
\langle\mathbf{x}-m(\mathbf{x}) \mathbf{e}, \mathbf{y}-m(\mathbf{y}) \mathbf{e}\rangle \leq\|\mathbf{x}-m(\mathbf{x}) \mathbf{e}\|_{2}\|\mathbf{y}-m(\mathbf{y}) \mathbf{e}\|_{2}
$$

with equality if and only if (2.14) holds. Thus,

$$
\|(1-t)(\mathbf{x}-m(\mathbf{x}) \mathbf{e})+t(\mathbf{y}-m(\mathbf{y}) \mathbf{e})\|_{2} \leq(1-t)\|\mathbf{x}-m(\mathbf{x}) \mathbf{e}\|_{2}+t\|\mathbf{y}-m(\mathbf{y}) \mathbf{e}\|_{2}
$$

with equality if and only if (2.14) holds. Finally, from (2.15) and (2.18), the lemma follows.

Lemma 2.4. For $\mathbf{x}, \mathbf{y} \in \mathscr{E}-\{\mathbf{e}\}$,

$$
f(\mathbf{x}) \geq\langle\nabla f(\mathbf{y}), \mathbf{x}\rangle
$$

with equality if and only if (2.14) holds for some $\alpha>0$.

Proof. $\mathscr{E}$ is a convex subset of $\mathscr{C}$ and $f$ is a convex function on $\mathscr{E}$. Moreover, $f$ is a differentiable function on $\mathscr{E}-\{\mathbf{e}\}$. Let $\mathbf{x}, \mathbf{y} \in \mathscr{E}-\{\mathbf{e}\}$. For all $t \in[0,1]$,

$$
f(t \mathbf{x}+(1-t) \mathbf{y}) \leq t f(\mathbf{x})+(1-t) f(\mathbf{y})
$$

Thus, for $0<t \leq 1$,

$$
\frac{f(\mathbf{y}+t(\mathbf{x}-\mathbf{y}))-f(\mathbf{y})}{t} \leq f(\mathbf{x})-f(\mathbf{y})
$$

Letting $t \rightarrow 0^{+}$yields

$$
\lim _{t \rightarrow 0^{+}} \frac{f(\mathbf{y}+t(\mathbf{x}-\mathbf{y}))-f(\mathbf{y})}{t}=\langle\nabla f(\mathbf{y}), \mathbf{x}-\mathbf{y}\rangle \leq f(\mathbf{x})-f(\mathbf{y}) .
$$

Hence,

$$
f(\mathbf{x})-f(\mathbf{y}) \geq\langle\nabla f(\mathbf{y}), \mathbf{x}\rangle-\langle\nabla f(\mathbf{y}), \mathbf{y}\rangle .
$$

Now, we use the fact that $\langle\nabla f(\mathbf{y}), \mathbf{y}\rangle=f(\mathbf{y})$ to conclude that

$$
f(\mathbf{x}) \geq\langle\nabla f(\mathbf{y}), \mathbf{x}\rangle .
$$

The equality in all the above inequalities holds if and only if $\mathbf{x}-a(\mathbf{x}) \mathbf{e}=\alpha(\mathbf{y}-m(\mathbf{y}) \mathbf{e})$ for some $\alpha \geq 0$. 
Corollary 2.5. For $\mathbf{x} \in \mathscr{E}-\{\mathbf{e}\}$,

$$
f(\mathbf{x}) \geq\left\langle\nabla f\left(\mathbf{x}^{2}\right), \mathbf{x}\right\rangle
$$

where $\nabla f\left(\mathbf{x}^{2}\right)$ is the gradient of $f$ with respect to $\mathbf{x}$ evaluated at $\mathbf{x}^{2}$. The equality in (2.25) holds if and only if $\mathbf{x}$ is one of the following convex combinations:

$$
\mathbf{x}_{i}(t)=t \mathbf{e}+(1-t) \mathbf{v}_{i}, \quad i=1,2, \ldots, n-1, \text { some } t \in[0,1) .
$$

Proof. Let $\mathbf{x}=\left(1, x_{2}, x_{3}, \ldots, x_{m}\right) \in \mathscr{E}-\{\mathbf{e}\}$. Then, $\mathbf{x}^{2} \in \mathscr{E}-\{\mathbf{e}\}$. Using Lemma 2.4, we obtain

$$
f(\mathbf{x}) \geq\left\langle\nabla f\left(\mathbf{x}^{2}\right), \mathbf{x}\right\rangle
$$

with equality if and only if

$$
\mathbf{x}-m(\mathbf{x}) \mathbf{e}=\alpha\left(\mathbf{x}^{2}-m\left(\mathbf{x}^{2}\right) \mathbf{e}\right)
$$

for some $\alpha \geq 0$. Thus, we have proved (2.25). In order to complete the proof, we observe that condition (2.28) is equivalent to

$$
\mathbf{x}-\alpha \mathbf{x}^{2}=m\left(\mathbf{x}-\alpha \mathbf{x}^{2}\right) \mathbf{e}
$$

for some $\alpha \geq 0$. Since $x_{1}=1,(2.29)$ is equivalent to

$$
1-\alpha=x_{2}-\alpha x_{2}^{2}=x_{3}-\alpha x_{3}^{2}=\cdots=x_{n}-\alpha x_{n}^{2}
$$

for some $\alpha \geq 0$. Hence, (2.28) is equivalent to (2.30).

Suppose that $(2.30)$ is true. If $\alpha=0$, then $1=x_{2}=\cdots=x_{n}$. This is a contradiction because $\mathbf{x} \neq \mathbf{e}$, thus $\alpha>0$.

If $x_{2}=0$, then $x_{3}=x_{4}=\cdots=x_{n}=0$, and thus $\mathbf{x}=\mathbf{v}_{1}$. Let $0<x_{2}<1$. Suppose $x_{3}<x_{2}$. From (2.30),

$$
\begin{gathered}
1-x_{2}=\alpha\left(1+x_{2}\right)\left(1-x_{2}\right), \\
x_{2}-x_{3}=\alpha\left(x_{2}+x_{3}\right)\left(x_{2}-x_{3}\right) .
\end{gathered}
$$

From these equations, we obtain $x_{3}=1$, which is a contradiction. Hence, $0<x_{2}<1 \mathrm{im}$ plies $x_{3}=x_{2}$. Now, if $x_{4}<x_{3}$, from $x_{2}=x_{3}$ and the equations

$$
\begin{gathered}
1-x_{2}=\alpha\left(1+x_{2}\right)\left(1-x_{2}\right), \\
x_{3}-x_{4}=\alpha\left(x_{3}+x_{4}\right)\left(x_{3}-x_{4}\right),
\end{gathered}
$$

we obtain $x_{4}=1$, which is a contradiction. Hence, $x_{4}=x_{3}$ if $0<x_{2}<1$. We continue in this fashion to conclude that $x_{n}=x_{n-1}=\cdots=x_{3}=x_{2}$. We have proved that $x_{1}=1$ and $0 \leq x_{2}<1$ imply that $\mathbf{x}=(1, t, \ldots, t)=t \mathbf{e}+(1-t) \mathbf{v}_{1}$ for some $t \in[0,1)$. Let $x_{2}=1$. 
8 Inequalities on the mean and standard deviation

If $x_{3}=0$, then $x_{4}=x_{5}=\cdots=x_{m}=0$, and thus $\mathbf{x}=\mathbf{v}_{2}$. Let $0<x_{3}<1$ and $x_{4}<x_{3}$. From (2.30),

$$
\begin{gathered}
1-x_{3}=\alpha\left(1+x_{3}\right)\left(1-x_{3}\right), \\
x_{3}-x_{4}=\alpha\left(x_{3}+x_{4}\right)\left(x_{3}-x_{4}\right) .
\end{gathered}
$$

From these equations, we obtain $x_{4}=1$, which is a contradiction. Hence, $0<x_{3}<1 \mathrm{im}$ plies $x_{4}=x_{3}$. Now, if $x_{5}<x_{4}$, from $x_{3}=x_{4}$ and the equations

$$
\begin{gathered}
1-x_{3}=\alpha\left(1+x_{3}\right)\left(1-x_{3}\right), \\
x_{4}-x_{5}=\alpha\left(x_{4}+x_{5}\right)\left(x_{4}-x_{5}\right),
\end{gathered}
$$

we obtain $x_{5}=1$, which is a contradiction. Therefore, $x_{5}=x_{4}$. We continue in this fashion to get $x_{n}=x_{n-1}=\cdots=x_{3}$. Thus, $x_{1}=x_{2}=1$, and $0 \leq x_{3}<1$ implies that $\mathbf{x}=(1,1, t, \ldots, t)$ $=t \mathbf{e}+(1-t) \mathbf{v}_{2}$ for some $t \in[0,1)$.

For $3 \leq k \leq n-2$, arguing as above, it can be proved that $x_{1}=x_{2}=\cdots=x_{k}=1$ and $0 \leq x_{k+1}<1$ implies that $\mathbf{x}=(1, \ldots, 1, t, \ldots, t)=t \mathbf{e}+(1-t) \mathbf{v}_{k}$. Finally, for $x_{1}=x_{2}=\cdots=$ $x_{n-1}=1$ and $0 \leq x_{n}<1$, we have $\mathbf{x}=t \mathbf{e}+\mathbf{v}_{n-1}$.

Conversely, if $\mathbf{x}$ is any of the convex combinations in (2.26), then (2.30) holds by choosing $\alpha=1 /(1+t)$.

Let us define the following optimization problem.

Problem 2.6. Let

$$
F: \mathbb{R}^{n} \longrightarrow \mathbb{R}
$$

be given by

$$
F(\mathbf{x})=f\left(\mathbf{x}^{2}\right)-(f(\mathbf{x}))^{2} .
$$

We want to find $\min _{\mathbf{x} \in \mathscr{C}} F(\mathbf{x})$. That is, find

$$
\min F(\mathbf{x})
$$

subject to the constraints

$$
\begin{gathered}
h_{1}(\mathbf{x})=x_{1}-1=0, \\
h_{i}(\mathbf{x})=x_{i}-x_{i-1} \leq 0, \quad 2 \leq i \leq n, \\
h_{n+1}(\mathbf{x})=-x_{n} \leq 0 .
\end{gathered}
$$

LEMma 2.7. (1) If $\mathbf{x} \in \mathscr{E}-\{\mathbf{e}\}$, then $\sum_{k=1}^{n} \partial_{k} F(\mathbf{x}) \leq 0$ with equality if and only if $\mathbf{x}$ is one of the convex combinations $\mathbf{x}_{k}(t)$ in (2.26).

(2) If $\mathbf{x}=\mathbf{x}_{N}(t)$ with $1 \leq N \leq n-2$, then

$$
\begin{gathered}
\partial_{1} F(\mathbf{x})=\cdots=\partial_{N} F(\mathbf{x})>0, \\
\partial_{N+1} F(\mathbf{x})=\cdots=\partial_{n} F(\mathbf{x})<0 .
\end{gathered}
$$


Proof. (1) The function $F$ has continuous first partial derivatives on $\mathscr{D}$, and for $\mathbf{x} \in \mathscr{D}$ and $1 \leq k \leq n$,

$$
\partial_{k} F(\mathbf{x})=2 x_{k} \partial_{k} f\left(\mathbf{x}^{2}\right)-2 f(\mathbf{x}) \partial_{k} f(\mathbf{x})
$$

By (2.9),

$$
\begin{aligned}
\sum_{k=1}^{n} \partial_{k} F(\mathbf{x}) & =2 \sum_{k=1}^{n} x_{k} \partial_{k} f\left(\mathbf{x}^{2}\right)-2 f(\mathbf{x}) \sum_{k=1}^{n} \partial_{k} f(\mathbf{x}) \\
& =2\left\langle\nabla f\left(\mathbf{x}^{2}\right), \mathbf{x}\right\rangle-2 f(\mathbf{x}) .
\end{aligned}
$$

It follows from Corollary 2.5 that $\sum_{k=1}^{n} \partial_{k} F(\mathbf{x}) \leq 0$ with equality if and only if $\mathbf{x}_{i}=t \mathbf{e}+$ $(1-t) \mathbf{v}_{i}, i=1, \ldots, n-1$.

(2) Let $\mathbf{x}=\mathbf{x}_{N}(t)$ with $1 \leq N \leq n-2$ fixed. Then, $\mathbf{x}=t \mathbf{e}+(1-t) \mathbf{v}_{N}$, some $t \in[0,1)$. Thus, $x_{1}=x_{2}=\cdots=x_{N}=1, x_{N+1}=x_{N+2}=\cdots=x_{n}=t$. From Theorem 1.1, $f(\mathbf{x})<1$. Moreover,

$$
\begin{aligned}
f(\mathbf{x})-m(\mathbf{x}) & =\sqrt{\frac{1}{n(n-1)}} \sqrt{N+(n-N) t^{2}-\frac{(N+(n-N) t)^{2}}{n}} \\
& =\sqrt{\frac{1}{n(n-1)}} \sqrt{\frac{n N+n(n-N) t^{2}-N^{2}-2 N(n-N) t-(n-N)^{2} t^{2}}{n}} \\
& =\frac{1}{n \sqrt{n-1}} \sqrt{N(n-N)(1-t) .}
\end{aligned}
$$

Replacing this result in (2.8), we obtain

$$
\begin{aligned}
\partial_{1} f(\mathbf{x}) & =\partial_{2} f(\mathbf{x})=\cdots=\partial_{N} f(\mathbf{x}) \\
& =\frac{1}{n}+\frac{1}{n(n-1)} \frac{1-m(\mathbf{x})}{f(\mathbf{x})-m(\mathbf{x})} \\
& =\frac{1}{n}+\frac{1}{\sqrt{n-1}} \frac{1-(N+(n-N) t) / n}{\sqrt{N(n-N)}(1-t)} \\
& =\frac{1}{n}+\frac{1}{\sqrt{n-1} n} \frac{\sqrt{n-N}}{\sqrt{N}}>0
\end{aligned}
$$

Similarly,

$$
\begin{aligned}
f\left(\mathbf{x}^{2}\right)-m\left(\mathbf{x}^{2}\right) & =\frac{1}{n \sqrt{n-1}} \sqrt{N(n-N)}\left(1-t^{2}\right), \\
\partial_{1} f\left(\mathbf{x}^{2}\right) & =\partial_{2} f\left(\mathbf{x}^{2}\right)=\cdots=\partial_{N} f\left(\mathbf{x}^{2}\right) \\
& =\frac{1}{n}+\frac{1}{n \sqrt{n-1}} \frac{\sqrt{n-N}}{\sqrt{N}}>0 .
\end{aligned}
$$


10 Inequalities on the mean and standard deviation

Therefore,

$$
\begin{aligned}
\partial_{1} F(\mathbf{x}) & =\partial_{2} F(\mathbf{x})=\cdots=\partial_{N} F(\mathbf{x}) \\
& =2 \partial_{1} f\left(\mathbf{x}^{2}\right)-2 f(\mathbf{x}) \partial_{1} f(\mathbf{x})=2(1-f(\mathbf{x})) \partial_{1} f(\mathbf{x})>0 .
\end{aligned}
$$

We have thus proved (2.39). We easily see that

$$
\partial_{N+1} F(\mathbf{x})=\partial_{N+2} F(\mathbf{x})=\cdots=\partial_{n} F(\mathbf{x}) .
$$

We have $\sum_{k=1}^{n} \partial_{k} F(\mathbf{x})=0$. Hence,

$$
\sum_{k=N+1}^{n} \partial_{k} F(\mathbf{x})=(n-N) \partial_{N+1} F(\mathbf{x})=-\sum_{k=1}^{N} \partial_{k} F(\mathbf{x})<0 .
$$

Thus, (2.40) follows.

We recall the following necessary condition for the existence of a minimum in nonlinear programming.

Theorem 2.8 (see [1, Theorem 9.2-4(1)]). Let $J: \Omega \subseteq V \rightarrow \mathbb{R}$ be a function defined over an open, convex subset $\Omega$ of a Hilbert space $V$ and let

$$
U=\left\{\mathbf{v} \in \Omega: \varphi_{i}(\mathbf{v}) \leq 0,1 \leq i \leq m\right\}
$$

be a subset of $\Omega$, the constraints $\varphi_{i}: \Omega \rightarrow \mathbb{R}, 1 \leq i \leq m$, being assumed to be convex. Let $\mathbf{u} \in U$ be a point at which the functions $\varphi_{i}, 1 \leq i \leq m$, and $J$ are differentiable. If the function $J$ has at $\mathbf{u}$ a relative minimum with respect to the set $U$ and if the constraints are qualified, then there exist numbers $\lambda_{i}(\mathbf{u}), 1 \leq i \leq m$, such that the Kuhn-Tucker conditions

$$
\begin{gathered}
\nabla J(\mathbf{u})+\sum_{i=1}^{m} \lambda_{i}(\mathbf{u}) \nabla \varphi_{i}(\mathbf{u})=\mathbf{0}, \\
\lambda_{i}(\mathbf{u}) \geq 0, \quad 1 \leq i \leq m, \quad \sum_{i=1}^{m} \lambda_{i}(\mathbf{u}) \varphi_{i}(\mathbf{u})=0
\end{gathered}
$$

are satisfied.

The convex constraints $\varphi_{i}$ in the above necessary condition are said to be qualified if either all the functions $\varphi_{i}$ are affine and the set $U$ is nonempty, or there exists a point $\mathbf{w} \in \Omega$ such that for each $i, \varphi_{i}(\mathbf{w}) \leq 0$ with strict inequality holding if $\varphi_{i}$ is not affine.

The solution to Problem 2.6 is given in the following theorem.

Theorem 2.9. One has

$$
\min _{\mathbf{x} \in \mathscr{\mathscr { C }}} F(\mathbf{x})=0=F(1,1,1, \ldots, 1, t)
$$

for any $t \in[0,1]$. 
Proof. We observe that $\mathscr{E}$ is a compact set and $F$ is a continuous function on $\mathscr{E}$. Then, there exists $\mathbf{x}_{0} \in \mathscr{E}$ such that $F\left(\mathbf{x}_{0}\right)=\min _{\mathbf{x} \in \mathscr{E}} F(\mathbf{x})$. The proof is based on the application of the necessary condition given in the preceding theorem. In Problem 2.6, we have $\Omega=V=\mathbb{R}^{n}$ with the inner product $\langle\mathbf{x}, \mathbf{y}\rangle=\sum_{k=1}^{n} x_{k} y_{k}, \varphi_{i}(\mathbf{x})=h_{i}(\mathbf{x}), 1 \leq i \leq n+1, U=$ $\mathscr{E}$ and $J=F$. The functions $h_{i}, 2 \leq i \leq n+1$, are linear. Therefore, they are convex and affine. In addition, the function $h_{1}(\mathbf{x})=x_{1}-1$ is affine and convex and $\mathscr{E}$ is nonempty. Consequently, the functions $h_{i}, 1 \leq i \leq n+1$, are qualified. Moreover, these functions and the objective function $F$ are differentiable at any point in $\mathscr{E}-\{\mathbf{e}\}$. The gradients of the constraint functions are

$$
\begin{gathered}
\nabla h_{1}(\mathbf{x})=(1,0,0,0, \ldots, 0)=\mathbf{e}_{1}, \\
\nabla h_{2}(\mathbf{x})=(-1,1,0,0, \ldots, 0), \\
\nabla h_{3}(\mathbf{x})=(0,-1,1,0, \ldots, 0), \\
\vdots \\
\nabla h_{n-1}(\mathbf{x})=(0,0, \ldots, 0,-1,1,0), \\
\nabla h_{n}(\mathbf{x})=(0,0, \ldots, 0,-1,1), \\
\nabla h_{n+1}(\mathbf{x})=(0,0, \ldots, 0,-1) .
\end{gathered}
$$

Suppose that $F$ has a relative minimum at $\mathbf{x} \in \mathscr{E}-\{\mathbf{e}\}$ with respect to the set $\mathscr{E}$. Then, there exist $\lambda_{i}(\mathbf{x}) \geq 0$ (for brevity $\left.\lambda_{i}=\lambda_{i}(\mathbf{x})\right), 1 \leq i \leq n+1$, such that the Kuhn-Tucker conditions

$$
\begin{gathered}
\nabla F(\mathbf{x})+\sum_{i=1}^{n+1} \lambda_{i} \nabla h_{i}(\mathbf{x})=\mathbf{0}, \\
\sum_{i=1}^{n+1} \lambda_{i} h_{i}(\mathbf{x})=0
\end{gathered}
$$

hold. Hence,

$$
\begin{gathered}
\nabla F(\mathbf{x})+\left(\lambda_{1}-\lambda_{2}, \lambda_{2}-\lambda_{3}, \lambda_{3}-\lambda_{4}, \ldots, \lambda_{n}-\lambda_{n+1}\right)=\mathbf{0}, \\
\lambda_{2}\left(x_{2}-1\right)+\lambda_{3}\left(x_{3}-x_{2}\right)+\cdots+\lambda_{n}\left(x_{n}-x_{n-1}\right)+\lambda_{n+1}\left(-x_{n}\right)=0 .
\end{gathered}
$$

From (2.55), as $\lambda_{i} \geq 0,1 \leq i \leq n+1$, and $0 \leq x_{n} \leq x_{n-1} \leq \cdots \leq x_{2} \leq 1$, we have

$$
\lambda_{k}\left(x_{k-1}-x_{k}\right)=0, \quad 2 \leq k \leq n, \quad \lambda_{n+1} x_{n}=0 .
$$

Now, from (2.54),

$$
\sum_{k=1}^{n} \partial_{k} F(\mathbf{x})+\lambda_{1}-\lambda_{n+1}=0 .
$$

We will conclude that $\lambda_{1}=0$ by showing that the cases $\lambda_{1}>0, x_{n}>0$ and $\lambda_{1}>0, x_{n}=0$ yield contradictions. 
12 Inequalities on the mean and standard deviation

Suppose $\lambda_{1}>0$ and $x_{n}>0$. In this case, $\lambda_{n+1} x_{n}=0$ implies $\lambda_{n+1}=0$. Thus, (2.57) becomes

$$
\sum_{k=1}^{n} \partial_{k} F(\mathbf{x})=-\lambda_{1}<0 .
$$

We apply Lemma 2.7 to conclude that $\mathbf{x}$ is not one of the convex combinations in (2.26). From (2.4),

$$
\begin{aligned}
\mathbf{x}= & \left(1-x_{2}\right) \mathbf{v}_{1}+\left(x_{2}-x_{3}\right) \mathbf{v}_{2}+\left(x_{3}-x_{4}\right) \mathbf{v}_{3} \\
& +\cdots+\left(x_{n-2}-x_{n-1}\right) \mathbf{v}_{n-2}+\left(x_{n-1}-x_{n}\right) \mathbf{v}_{n-1}+x_{n} \mathbf{v}_{n}
\end{aligned}
$$

Then, there are at least two indexes $i, j$ such that

$$
1=\cdots=x_{i}>x_{i+1}=\cdots=x_{j}>x_{j+1} .
$$

Therefore,

$$
\begin{gathered}
\partial_{1} F(\mathbf{x})=\cdots=\partial_{i} F(\mathbf{x}), \\
\partial_{i+1} F(\mathbf{x})=\cdots=\partial_{j} F(\mathbf{x}) .
\end{gathered}
$$

From (2.56), we get $\lambda_{i+1}=0$ and $\lambda_{j+1}=0$. Now, from (2.54),

$$
\begin{gathered}
\partial_{i} F(\mathbf{x})=-\lambda_{i} \leq 0, \\
\partial_{i+1} F(\mathbf{x})=\lambda_{i+2} \geq 0, \\
\partial_{j} F(\mathbf{x})=-\lambda_{j} \leq 0, \\
\partial_{n} F(\mathbf{x})=-\lambda_{n} \leq 0 .
\end{gathered}
$$

The above equalities and inequalities together with (2.8) and (2.41) give

$$
\begin{aligned}
& \frac{1}{n}(1-f(\mathbf{x}))+\frac{1}{n(n-1)}\left(\frac{1-m\left(\mathbf{x}^{2}\right)}{f\left(\mathbf{x}^{2}\right)-m\left(\mathbf{x}^{2}\right)}-\frac{1-m(\mathbf{x})}{f(\mathbf{x})-m(\mathbf{x})}\right) \leq 0, \\
& \frac{1}{n}(1-f(\mathbf{x}))+\frac{1}{n(n-1)}\left(\frac{x_{j}^{2}-m\left(\mathbf{x}^{2}\right)}{f\left(\mathbf{x}^{2}\right)-m\left(\mathbf{x}^{2}\right)}-\frac{x_{j}-m(\mathbf{x})}{f(\mathbf{x})-m(\mathbf{x})}\right)=0, \\
& \frac{1}{n}(1-f(\mathbf{x}))+\frac{1}{n(n-1)}\left(\frac{x_{n}^{2}-m\left(\mathbf{x}^{2}\right)}{f\left(\mathbf{x}^{2}\right)-m\left(\mathbf{x}^{2}\right)}-\frac{x_{n}-m(\mathbf{x})}{f(\mathbf{x})-m(\mathbf{x})}\right) \leq 0 .
\end{aligned}
$$

Subtracting (2.64) from (2.63) and (2.65), we obtain

$$
\begin{aligned}
\frac{1-x_{j}^{2}}{f\left(\mathbf{x}^{2}\right)-m\left(\mathbf{x}^{2}\right)} & \leq \frac{1-x_{j}}{f\left(\mathbf{x}^{2}\right)-m\left(\mathbf{x}^{2}\right)}, \\
\frac{x_{n}^{2}-x_{j}^{2}}{f\left(\mathbf{x}^{2}\right)-m\left(\mathbf{x}^{2}\right)} & \leq \frac{x_{n}-x_{j}}{f\left(\mathbf{x}^{2}\right)-m\left(\mathbf{x}^{2}\right)} .
\end{aligned}
$$


Dividing these inequalities by $\left(1-x_{j}\right)$ and $\left(x_{n}-x_{j}\right)$, respectively, we get

$$
\begin{aligned}
& \frac{1+x_{j}}{f\left(\mathbf{x}^{2}\right)-m\left(\mathbf{x}^{2}\right)} \leq \frac{1}{f\left(\mathbf{x}^{2}\right)-m\left(\mathbf{x}^{2}\right)}, \\
& \frac{x_{n}+x_{j}}{f\left(\mathbf{x}^{2}\right)-a\left(\mathbf{x}^{2}\right)} \geq \frac{1}{f\left(\mathbf{x}^{2}\right)-a\left(\mathbf{x}^{2}\right)} .
\end{aligned}
$$

The last two inequalities imply $x_{n} \geq x_{j}$, which is contradiction.

Suppose now that $\lambda_{1}>0$ and $x_{n}=0$. Let $l$ be the largest index such that $x_{l}>0$. Thus, $x_{l+1}=0$. From (2.55),

$$
\lambda_{2}\left(x_{2}-1\right)+\lambda_{3}\left(x_{3}-x_{2}\right)+\cdots+\lambda_{l}\left(x_{l}-x_{l-1}\right)+\lambda_{l+1}\left(-x_{l}\right)=0 .
$$

Then,

$$
\lambda_{k}\left(x_{k-1}-x_{k}\right)=0, \quad 2 \leq k \leq l, \quad \lambda_{l+1} x_{l}=0 .
$$

Hence, $\lambda_{l+1}=0$. If $l=n-1$, then $\lambda_{n}=0$ and $\partial_{n} F(\mathbf{x})=\lambda_{n+1} \geq 0$. If $l \leq n-2$, then $\partial_{l} F(\mathbf{x})=$ $-\lambda_{l} \leq 0$. In both situations, we conclude that $\mathbf{x}$ is not one of the convex combinations in (2.26). Therefore, there are at least two indexes $i, j$ such that

$$
1=\cdots=x_{i}>x_{i+1}=\cdots=x_{j}>x_{j+1} .
$$

Now, we repeat the argument used above to get that $x_{l} \geq x_{j}$, which is a contradiction.

Consequently, $\lambda_{1}=0$. From (2.57),

$$
\sum_{k=1}^{n} \partial_{k} F(\mathbf{x})=\lambda_{n+1} \geq 0
$$

We apply now Lemma 2.7 to conclude that $\mathbf{x}$ is one of the convex combinations in (2.26). Let $\mathbf{x}=\mathbf{x}_{N}(t)=t \mathbf{e}+(1-t) \mathbf{v}_{N}, 1 \leq N \leq n-2$, and $t \in[0,1)$. Then, $x_{1}=x_{2}=\cdots=x_{N}=1$, $x_{N+1}=x_{N+2}=\cdots=x_{n}=t$, and $h_{N+1}(\mathbf{x})=t-1<0$. From (2.56), we obtain $\lambda_{N+1}=0$. Thus, from (2.54), $\partial_{N+1} F(\mathbf{x})=\lambda_{N+2} \geq 0$. This contradicts (2.40). Thus, $\mathbf{x} \neq \mathbf{x}_{N}(t)$ for $N=$ $1,2, \ldots, n-2$ and $t \in[0,1)$. Consequently, $\mathbf{x}=\mathbf{x}_{n-1}(t)=(1,1, \ldots, 1, t)$ for some $t \in[0,1)$.

Finally,

$$
F(1,1, \ldots, 1, t)=f\left(1,1, \ldots, 1, t^{2}\right)-(f(1,1, \ldots, 1, t))^{2}=1-1=0
$$

for any $t \in[0,1]$. Hence, $\min _{\mathbf{x} \in \mathscr{C}} F(\mathbf{x})=0=F(1,1, \ldots, 1, t)$ for any $t \in[0,1]$. Thus, the theorem has been proved.

Theorem 2.10. If $y_{1} \geq y_{2} \geq y_{3} \geq \cdots \geq y_{n} \geq 0$, then

$$
m\left(\mathbf{y}^{2^{p}}\right)+\frac{1}{\sqrt{n-1}} s\left(\mathbf{y}^{2^{p}}\right) \leq \sqrt{m\left(\mathbf{y}^{2^{p+1}}\right)+\frac{1}{\sqrt{n-1}} s\left(\mathbf{y}^{2^{p+1}}\right)},
$$


14 Inequalities on the mean and standard deviation

that is,

$$
\begin{aligned}
& \frac{\sum_{k=1}^{n} y_{k}^{2^{p}}}{n}+\frac{1}{\sqrt{n(n-1)}} \sqrt{\sum_{k=1}^{n} y_{k}^{2^{p+1}}-\frac{\left(\sum_{k=1}^{n} y_{k}^{2^{p}}\right)^{2}}{n}} \\
& \quad \leq\left[\frac{\sum_{k=1}^{n} y_{k}^{2^{p+1}}}{n}+\frac{1}{\sqrt{n(n-1)}} \sqrt{\sum_{k=1}^{n} y_{k}^{2^{p+2}}-\frac{\left(\sum_{k=1}^{n} y_{k}^{2^{p+1}}\right)^{2}}{n}}\right]^{1 / 2}
\end{aligned}
$$

for $p=0,1,2, \ldots$. The equality holds if and only if $y_{1}=y_{2}=\cdots=y_{n-1}$.

Proof. If $y_{1}=0$, then $y_{2}=y_{3}=\cdots=y_{n}=0$ and the theorem is immediate. Hence, we assume that $y_{1}>0$. Let $p$ be a nonnegative integer and let $x_{k}=y_{k} / y_{1}$ for $k=1,2, \ldots, n$. Clearly, $1=x_{1}^{2^{p}} \geq x_{2}^{2^{p}} \geq x_{3}^{2^{p}} \geq \cdots \geq x_{n}^{2^{p}} \geq 0$. From Theorem 2.9, we have

$$
\left(f\left(1, x_{2}^{2^{p}}, x_{3}^{2^{p}}, \ldots, x_{m}^{2^{p}}\right)\right)^{2} \leq f\left(1, x_{2}^{2^{p+1}}, x_{3}^{2^{p+1}}, \ldots, x_{m}^{2^{p+1}}\right)
$$

that is,

$$
\begin{aligned}
& \left(\frac{1+\sum_{k=2}^{n} x_{k}^{2^{p}}}{n}+\frac{1}{\sqrt{n(n-1)}} \sqrt{\left.1+\sum_{k=2}^{n} x_{k}^{2^{p+1}}-\frac{\left(1+\sum_{j=2}^{n} x_{j}^{2^{p}}\right)^{2}}{n}\right)^{2}}\right. \\
& \leq \frac{1+\sum_{k=2}^{n} x_{k}^{2^{p+1}}}{n}+\frac{1}{\sqrt{n(n-1)}} \sqrt{1+\sum_{k=2}^{n} x_{k}^{2^{p+2}}-\frac{\left(1+\sum_{j=2}^{n} x_{j}^{2^{p+1}}\right)^{2}}{n}}
\end{aligned}
$$

with equality if and only if $x_{1}=x_{2}=\cdots=x_{n-1}$. Multiplying by $y_{1}^{2^{p+1}}$, the inequality in (2.74) is obtained with equality if and only if $y_{1}=y_{2}=\cdots=y_{n-1}$. This completes the proof.

Corollary 2.11. Let $y_{1} \geq y_{2} \geq y_{3} \geq \cdots \geq y_{n} \geq 0$. Then $\left(l_{2^{p}}(\mathbf{y})\right)_{p=0}^{\infty}$,

$$
\begin{aligned}
l_{2^{p}}(\mathbf{y}) & =\left(\frac{\|\mathbf{y}\|_{2^{p}}^{2^{p}}}{n}+\frac{1}{\sqrt{n(n-1)}} \sqrt{\|y\|_{2^{p+1}}^{2^{p+1}}-\frac{\|\mathbf{y}\|_{2^{p}}^{2^{p+1}}}{n}}\right)^{2^{-p}} \\
& =\left(m\left(\mathbf{y}^{2^{p}}\right)+\frac{1}{\sqrt{n-1}} s\left(\mathbf{y}^{2^{p}}\right)\right)^{2^{-p}},
\end{aligned}
$$

is an strictly increasing sequence converging to $y_{1}$ except if $y_{1}=y_{2}=\cdots=y_{n-1}$. In this case, $l_{2^{p}}(\mathbf{y})=y_{1}$ for all $p$. 
Proof. We know that $\left(l_{2^{p}}(\mathbf{y})\right)_{p=0}^{\infty}$ is a sequence of lower bounds for $y_{1}$. From Theorem 2.1, this sequence converges to $y_{1}$. Applying inequality (2.74), we obtain

$$
\begin{aligned}
& \left(\frac{\sum_{k=1}^{n} y_{k}^{2^{p}}}{n}+\frac{1}{\sqrt{n(n-1)}} \sqrt{\left.\sum_{k=1}^{n} y_{k}^{2^{p+1}}-\frac{\left(\sum_{j=1}^{n} y_{j}^{2^{p}}\right)^{2}}{n}\right)^{2}}\right. \\
& \quad \leq \frac{\sum_{k=1}^{n} y_{k}^{2^{p+1}}}{n}+\frac{1}{\sqrt{n(n-1)}} \sqrt{\sum_{k=1}^{n} y_{k}^{2^{p+2}}-\frac{\left(\sum_{j=1}^{n} y_{j}^{2^{p+1}}\right)^{2}}{n}} .
\end{aligned}
$$

Therefore, $l_{2^{p}}^{2^{p+1}}(\mathbf{y}) \leq l_{2^{p+1}}^{2^{p+1}}(\mathbf{y})$, that is, $l_{2^{p}}(\mathbf{y}) \leq l_{2^{p+1}}(\mathbf{y})$. The equality in all the above inequalities takes place if and only if $\lambda_{1}=y_{2}=\cdots=y_{n-1}$. In this case, $l_{2^{p}}(\mathbf{y})=\lambda_{1}$ for all $p$.

\section{Acknowledgment}

This work is supported by Fondecyt 1040218, Chile.

\section{References}

[1] P. G. Ciarlet, Introduction to Numerical Linear Algebra and Optimisation, Cambridge Texts in Applied Mathematics, Cambridge University Press, Cambridge, 1991.

[2] O. Rojo and H. Rojo, A decreasing sequence of upper bounds on the largest Laplacian eigenvalue of a graph, Linear Algebra and Its Applications 381 (2004), 97-116.

[3] H. Wolkowicz and G. P. H. Styan, Bounds for eigenvalues using traces, Linear Algebra and Its Applications 29 (1980), 471-506.

Oscar Rojo: Departamento de Matemáticas, Universidad Católica del Norte, Casilla 1280, Antofagasta, Chile

E-mail address: orojo@ucn.cl 